Article

\title{
Sustaining Astronauts: Resource Limitations, Technology Needs, and Parallels between Spaceflight Food Systems and those on Earth
}

\author{
Grace L. Douglas ${ }^{1, *}$, Raymond M. Wheeler ${ }^{2}$ and Ralph F. Fritsche ${ }^{3}$ \\ 1 Human Health and Performance Directorate, Johnson Space Center, NASA, Houston, TX 77058, USA \\ 2 Exploration Research and Technology, Kennedy Space Center, NASA, Merritt Island, FL 32899, USA; \\ raymond.m.wheeler@nasa.gov \\ 3 Utilization and Life Sciences Office, Kennedy Space Center, NASA, Merritt Island, FL 32899, USA; \\ ralph.f.fritsche@nasa.gov \\ * Correspondence: grace.1.douglas@nasa.gov
}

check for updates

Citation: Douglas, G.L.; Wheeler, R.M.; Fritsche, R.F. Sustaining Astronauts: Resource Limitations, Technology Needs, and Parallels between Spaceflight Food Systems and those on Earth. Sustainability 2021, 13, 9424. https://doi.org/ $10.3390 /$ su13169424

Academic Editors: Ann Barrett and Michelle Richardson

Received: 29 June 2021

Accepted: 14 August 2021

Published: 22 August 2021

Publisher's Note: MDPI stays neutral with regard to jurisdictional claims in published maps and institutional affiliations.

Copyright: (c) 2021 by the authors. Licensee MDPI, Basel, Switzerland. This article is an open access article distributed under the terms and conditions of the Creative Commons Attribution (CC BY) license (https:/ / creativecommons.org/licenses/by/ $4.0 /)$.

\begin{abstract}
Food and nutrition are critical to health and performance and therefore the success of human space exploration. However, the shelf-stable food system currently in use on the International Space Station is not sustainable as missions become longer and further from Earth, even with modification for mass and water efficiencies. Here, we provide a potential approach toward sustainability with the phased addition of bioregenerative foods over the course of NASA's current mission plans. Significant advances in both knowledge and technology are still needed to inform nutrition, acceptability, safety, reliability, and resource and integration trades between bioregenerative and other food systems. Sustainability goals on Earth are driving similar research into bioregenerative solutions with the potential for infusion across spaceflight and Earth research that benefits both.
\end{abstract}

Keywords: sustainable; spaceflight; food; crops

\section{Introduction}

Food and nutrition are central to every aspect of health and performance. Throughout history, the adequacy of the food system has been critical to the success of human exploration $[1,2]$. The field of human nutrition has greatly advanced over the past hundred years, and we have established the requirements of a safe, nutritious, and acceptable food system. As we transition to the era of deep space exploration, it seems straightforward that we would apply this knowledge to future exploration food systems. However, resource constraints and shelf-life requirements on space vehicles can conflict with what seems on Earth to be basic and necessary food system solutions (e.g., cold storage), challenging food system provisioning as missions become longer and further from Earth.

Resource limitations include launch mass, volume, water, power, preparation equipment, and crew time, which has restricted the food system to date to mostly individually packaged, shelf-stable foods. Microgravity further restricts the system to one that does not produce a lot of crumbs and with foods that have viscosity and surface tension to contain them in the package or on the utensil during meal consumption. Preparation capability is limited to the addition of water or heat. The space food system has evolved from the tubes and cubes of the Mercury missions to the system used on the International Space Station today, which incorporates a variety of processed foods in lightweight laminate packaging. The specifics of that evolution have been reviewed previously [3-6], but the food system is still completely Earth-dependent. Space exploration and settlement on other planets ultimately will require a more sustainable food system that is less dependent on Earth.

Sustainability of a spaceflight food system has many parallels to the challenges of sustainability for Earth-based food systems, and there is potential for solutions that contribute to sustainability in spaceflight to simultaneously contribute on Earth. We are still far from 
a fully closed food system in spaceflight, as many of the necessary ecosystem components are not available. However, in order to understand the magnitude of this challenge, it is important to understand the resource challenges associated with spaceflight and how this has driven the current food system solutions. Here, we discuss how resource challenges were met throughout the history of the spaceflight food system, and considerations for a phased approach toward sustainability for those developing solutions for future exploration.

\section{Space Food System Resources-International Space Station}

The standard food system used for the 6-12 month missions on the International Space Station (ISS) includes around 200 foods stowed in categories, such as breakfast, vegetables and soups, and beverages. The foods are shared pantry-style by the astronauts in the United States Operating Segment, which includes US, Canadian Space Agency (CSA), European Space Agency (ESA), and Japanese Space Agency (JAXA) crew members. The Russian Cosmonauts are provided with a separate, Russian food system. The foods are either retort thermostabilized, freeze-dried, irradiated, or low to intermediate moisture. All beverages are powders packaged to enable the addition of water in microgravity. Food preparation is limited to the addition of metered hot or ambient temperature water, a conduction food warmer, and a small cold storage unit large enough to hold some condiments and beverages. Water is limited, and most of it is recycled [7]. Mealtimes, which are important psychosocially to crew gatherings, celebrations, and crew cohesion [8], are also restricted by available time, which is largely dedicated to science and maintenance activities.

The current food system has been developed to provide the spaceflight nutritional requirements with as much variety and choice as possible within a closed mass and volume allotment. It is commonly assumed that high performers, such as astronauts, will consume whatever is required, regardless of acceptability, to successfully complete a mission. However, food and mealtime activities have been shown to become more important with length of isolation and confinement [9]. If food is not well liked, it is under-consumed, which may result in nutritional inadequacy with the closed food system in spaceflight. Therefore, food acceptability is as important as nutrient content. Weight loss has been common on most spaceflight missions to date, and multiple factors may contribute [6]. Variety, choice, and ease of preparation are commonly identified by crew as important factors to an acceptable food system. The logistics of a mass and volume constrained system make it a challenge to increase variety further. Adding foods means other foods would have to be removed, which has as much potential to reduce the availability of favorite foods as to increase them.

Providing food to each astronaut completely according to preference is not possible on ISS because late crew changes and resupply delays impact the schedule alignment of preference foods with the intended crew in orbit. However, a limited set of preference foods and beverages are provisioned to augment the standard food set. Additionally, resupply vehicles, which have increased in number with the launches of commercial vehicles in recent years, bring extra foods, including some fresh foods such as apples and oranges, extra shelf-stable preference foods, and occasionally some frozen (ice cream) or refrigerated foods (cheeses). The preference and extra foods can provide $12-25 \%$ of the foods consumed on ISS [6].

\section{Space Food System Limitations-Considerations for Exploration}

Issues that need to be addressed for any food system under consideration for long duration, deep space exploration missions have been reviewed previously, and they include requirements for nutrition, acceptability, safety, shelf life, reliability, resources, and human factors [10]. No food system has yet been identified that will meet all these requirements.

The current ISS shelf-stable food system is the only established, reliable option to date, but some nutrients and quality factors degrade during room temperature storage to unacceptable levels within 1 to 3 years, which varies by food [11,12]. Exploration missions to Mars, especially those with prepositioned foods, may require nutritional and quality stability for 5 years. Cold storage has the potential to improve this shelf life, but to what 
extent is not yet quantified, and this would increase an already overburdened resource requirement. Even if passive refrigeration solutions were developed that could use the spaceflight environment, resources would be needed for continued protection from the vacuum of space and the fluctuation in temperature, and resource trades compared to active refrigeration are not yet quantified.

Bioregenerative food systems, which include the growing of crops and any other system that would continue to produce new food from resources available during the mission, still require significant research, development, and system integration before they can be incorporated reliably into a spaceflight food system. Even when technologies may benefit both Earth and spaceflight, system requirements may be different. There are new challenges not encountered on Earth such as exposure to radiation, operations at reduced pressures, and function in microgravity and partial gravity environments.

Many bioregenerative foods that are currently in development or production on Earth, such as algae and cell culture, are bioreactor products. These require extensive processing, preparation, system experts with dedicated time, and infrastructure that would introduce vehicle integration challenges. Most studies that present these foods as spaceflight solutions do not discuss these challenges $[13,14]$, do not acknowledge the history of work in the area in relation to spaceflight, and do not provide solutions to overcome the poor acceptability of many of these products to date [15-17], leaving a substantial gap.

Animal protein is another bioregenerative food that may be an important contribution for both nutrition and acceptability over time [18,19], as a vegan-only system would not be acceptable to many astronauts. Although there has been recent progress in animal cell culture, substantial infrastructure efficiencies and culture media sustainability solutions would need to be achieved for spaceflight feasibility [20]. Farming live animals or fish would present an entirely different set of challenges that include feed, waste removal systems, processing, and welfare in novel environments that are not resource realistic for any currently planned mission. Insects have also been proposed [21], but they present acceptability challenges in addition to resource and processing challenges. Astronauts who want to consume animal protein will continue to rely on prepackaged sources until advances are achieved. Advances in bioregenerative technologies for Earth may eventually enable the integration of some of these solutions into spaceflight vehicles, and limited spaceflight resources may drive further advances in efficiency.

Bioregenerative foods also bring with them a new risk of food scarcity if the expected nutritional output is not achieved [22]. Finally, there are the safety challenges that must be considered. Food safety must be established in any system prior to use as a supplementary food system. The significant resources required to confirm the safety of shelf-stable foods on Earth would not be transferrable to spaceflight. Testing capabilities would either need to advance or extremely reliable protocols would need to be developed [23]. A foodborne illness may be worse in spaceflight where medical capabilities and supplies may be more limited and microgravity may increase spread.

Despite these challenges, the success of future space exploration will rely on the successful development of bioregenerative, sustainable systems that are less dependent on Earth and trade favorably in nutrition, acceptability, safety, resources, and integration with the spacecraft against current systems.

A few concepts need to be defined at this point. First, we need to define a "sustainable spaceflight food system". A commonly used definition for sustainable development is development that meets the needs of the present without compromising the needs of the future generations [24]. Space exploration supports Earth sustainability with the potential for exploration and settlements on other planetary surfaces but only in the case that those settlements are less dependent on Earth. Therefore, a "sustainable spaceflight food system" must be truly bioregenerative, and we propose to define it as "a food system where all resources and infrastructure are produced in situ and sustainably with either no resupply, or minimal resupply sustainable within Earth's resources". 
Sustainability would include the use of in situ resource utilization (ISRU) to produce equipment, maintenance tools, and spare parts, although this would require additional infrastructure that is not reviewed here. Further work would similarly be needed to identify where ISRU systems would become more cost effective than bringing supplies from Earth Important questions remain as to the feasibility of generating resources on another surface, which could improve the sustainability of a bioregenerative food system infrastructure. For example, even if methane or other organic compounds can be produced from $\mathrm{CO}_{2}$ and used by microorganisms to produce biopolymers that can then be used for 3D printing [25], further work would be needed to identify where this system would become more cost effective than bringing supplies from Earth. Ground research is still critical to understand some of these aspects further prior to spaceflight testing.

Second, we need to define the mission. Food systems may vary depending on the phase of the mission (transit or surface), the length of the mission (months or years), the destination (moon or Mars), the goals (science and exploration or settlement), the resources (crew time, mass, volume, power, water, etc.), and the number of crew. For example, on the transit phase of an exploration mission, a small crew size may have time for some extra food-related activities that fit within the resources available, but once they arrive at a surface setting, they may need to spend most of their time on science and exploration.

Third, we need to determine the length of missions where it is more cost effective to bring infrastructure and resources for growing and processing food than to bring the current shelf-stable food system. This may vary depending on the bioregenerative infrastructure. We suggest that for this purpose, food systems should be considered modular, with the potential to be integrated at increasing percentages. This concept would require knowledge of specific flight-ready infrastructure and integration requirements, which is not currently available beyond proof of concept scale equipment [26]. As mentioned, bioregenerative food systems all still require significant ground-based research and development before we have the knowledge about their resources, integration impacts, and infrastructure requirements to make accurate trades in comparison to the current shelfstable spaceflight food system. Promising candidates from ground experiments will require demonstration, integration, and validation on a space vehicle or habitat to confirm resource and infrastructure data. Near-term exploration missions will provide the platforms to conduct this research in relevant environments.

\section{Food Systems on Near-Term Exploration Missions}

\subsection{Initial Lunar Exploration}

Initial exploration to the lunar surface will likely have small crews of 4-6 with a primary focus on exploration and science. There likely will be no food specialists, and the main mission tasks of exploration, system maintenance, and science will take up most crew time. Food systems will need to be the type that most people on Earth would want to use every day in their kitchens after a long day of work. These missions will be short in duration (less than 3 months). The infrastructure required to produce food would far outweigh the resource cost of taking a shelf-stable food system, and the timeframe will be too short to grow anything substantial. Mass may be very restricted on these missions, and in some scenarios, food systems may be prepositioned, and resupply will not be possible.

These missions are likely to continue using the shelf-stable prepackaged foods, with an emphasis on determining methods within this system to reduce mass. Strategies may include increasing nutrient density through an increase in fat content or reduction in water or changes in packaging design. Any changes need to be structured within nutritional requirements, water recycling capabilities, and overall food provisioning. For instance, although saturated fat is more stable to oxidation, it has lower limits for health. Most missions require food to have a multi-year shelf life, which may be a challenge to meet with some unsaturated fats. However, there is currently debate over the health impacts of saturated fat, based on dietary components providing the saturated fat, and this may impact product development requirements for future high-density food options [27,28]. 
Nutrient-dense meal replacement bars, which may both increase fat content and reduce the water content of the food system, face acceptability limitations. This challenge was highlighted by recent results from a ground analog 30-day mission simulation, where one meal a day was replaced with nutrient-dense meal replacement bars that were intended to reduce food system mass [29]. Despite acceptable scores in sensory evaluations, the use of meal replacement bars over the short duration simulations was associated with reduced caloric intake, more weight loss, and decrements in mood and neurobehavioral functioning, with variety and monotony as contributing factors [29]. This type of mass-reduction solution may be feasible for short duration missions, but more work would be needed to determine the full health and performance impacts and acceptable implementation strategies. Similarly, any savings from reductions in the water content of the food are dependent on mission length, water recycling capability, and water needs for systems other than food [30].

Initial lunar exploration may provide an opportunity for those working on promising bioregenerative ground experiments to conduct early research in the deep space radiation environment simultaneously with microgravity at NASA's planned Gateway or with partial gravity on the lunar surface. The response of seeds, plants, algae, fungi, and microbes to these new stressors when coupled with other more familiar spaceflight stressors has yet to be demonstrated. Opportunities to conduct research in the deep space environment will be highly competitive, and biological experiments will compete against a range of research from other physical science disciplines. As a result, researchers need to be prepared to take advantage of any opportunities that may become available whether they be at the Gateway or another Artemis vehicle infrastructure.

\subsection{Early Lunar Outposts and Demonstration of Capabilities}

Early lunar establishment may still require all supplies to be brought from Earth, including shelf-stable foods. However, as capabilities expand, these outposts can provide a testbed to continue bioregenerative food production work from Earth [31-35] and the ISS $[26,35]$ under the unique environmental conditions of the deep space radiation and partial gravity environment.

Initial activities may supplement the prepackaged food system with bioregenerative foods. Food preparation infrastructure is expected to continue to be limited at these outposts. Bioregenerative foods, which can be demonstrated to be both safe and palatable with no additional processing prior to consumption (e.g., salad crops), would be ideal initial candidates.

System failures would be expected as techniques and systems are validated, and crew would not yet rely on the bioregenerative products. Hardware reliability and failure modes need to be characterized and well understood with systems designed for repair versus replacement. Ground work in this area at places such as the EDEN-ISS facility in the Antarctic [36] will provide the baseline to establish techniques and hardware for validation in spaceflight.

During this time, testing may begin with novel yet sustainable food production methods that show promise in ground experiments and acceptability over time by crews in ground analogs. These systems may leverage solutions in development to meet the challenge of sustainably feeding a growing population on Earth, where food scarcity and food security represent a significant issue. It is important to recognize that many food products would not be acceptable for consumption as is (e.g., algae, fermentation products) [15]. Some systems may require specialists, the development and maintenance of processing or preparation equipment, or full system automation to produce acceptable and safe products. All equipment and systems would similarly need to be compatible with altered gravity and pressure and high levels of radiation. Technologies would be evaluated first on the ground for factors such as growth materials, power consumption, the need for spares and tools for system maintenance, integration with other vehicle systems such as air and water, crew time, and the acceptability of both process and products to crew when 
incorporated into mission-relevant schedules. These factors would enable assessment of feasibility and comparative trades with other food system options.

Some food production technologies that are viable on Earth use extensive resources that cannot be directly transferred to the spaceflight environment, including time for production and maintenance, and methods for processing, cleaning, and confirming food safety. Viable food production technologies will need to sustain crew with fewer resources. Similar to Earth, food production systems will impact the health of the spacecraft environment but on a much faster timeline. Mitigations will need to occur more efficiently in these early small habitats.

In much the same way that NASA's planned Artemis missions to the Moon will serve as an analog for future surface operations on Mars, they may serve as an analog for the development of future bioregenerative food production systems.

\subsection{Initial Mars Missions}

Early Mars missions may only have four to six crew members. The primary focus of these missions may be to conduct exploration and science with the goal of eventual expansion of surface presence and capabilities. For initial Mars missions, food may need to be prepositioned on the Martian surface. Such a scenario cannot accommodate preferences for late crew assignments or changes, so the food system may be limited to a standard, generally acceptable, food set.

We anticipate that early Mars missions will benefit from supplemental bioregenerative food production methods established on ISS and lunar missions to provide additional, targeted nutrients as well as variety, flavor, and freshness that may degrade in the prepackaged food over time [11,12].

Similar to early lunar missions, early Mars missions will not bring food specialists who can spend hours preparing meals. Any near-term food system will need to be the type that most people on Earth would want to use every day in their kitchens after a long day of work. Ideally, they would require few resources, as food preparation capability is still likely to be limited. Although we do not yet know the details of an early Mars transit and surface food system, it may still contain a large portion of shelf-stable, readyto-eat meals. However, the evolvable and scalable systems developed on the ground and validated on the moon may now begin to support supplemental bioregenerative food systems on Mars, with increasing amounts of food grown in situ as surface durations increase, technologies advance, and the cost trades/production ratios favor the additional infrastructure and integration.

As exploration of the Moon and Mars continues and the development of novel food production methods matures, it is expected that some alternative food system concepts will become viable candidates for inclusion on future missions. The ability to grow different foods or process ingredients in spaceflight will serve to increase the variety of nutritious and acceptable foods available to crews. However, it is important to continue to emphasize that the expectation is that most if not all of these systems would require significant development and integration to become feasible for use in spaceflight.

\subsection{Exploration Settlements on the Moon and Mars}

As human settlements on other planetary surfaces expand beyond exploration, food production specialists may be among the first settlers, and systems will be able to diversify. The research we do now is critical to prove concepts, reduce resource requirements, and effectively integrate sustainable food production systems within the infrastructure and operations of the settlements artificial ecosystem. These systems may form an important component of a bioregenerative life support system where they may aid in atmospheric revitalization, waste management, and water treatment and thus help to ensure the survival of the settlement. Although full discussion of how foods fit into bioregenerative life support systems are out of scope here, we encourage anyone interested in bioregenerative food and life support systems to investigate the complexity of these systems, which has 
been reviewed elsewhere $[16,33,35,37,38]$. The assumptions and models used in many trade studies to date are useful but need updating and improvement [39-42]. Significant research and development on all of these systems is needed to inform actual resource requirements necessary for cost trades that will indicate when missions will favor the additional infrastructure and integration.

In the next section, we present food crops as a case study of what the path from lunar research to Mars surface settlement may look like for a new food production technology based on gaps known today.

\section{Introduction of In Situ Grown Foods-Food Crops as a Case Study}

\subsection{Current State of the Research}

A lot of work has been done with bioregenerative food systems over the past 60 years. We would encourage anyone working in these areas to investigate this past research, which exists for many concepts $[16,17,35]$. We are focusing on crops as an example of a roadmap to integration with the space food system for several reasons. First, crops are commonly considered acceptable to consume with minimal or no processing. Salad crops can provide a variety of colors, flavors, and textures, which along with some horticultural activities may contribute to psychosocial benefits in spaceflight $[4,43]$. Additionally, their production could provide important contributions to a sustainable food production and life support system, both as a waste user $\left(\mathrm{CO}_{2}\right.$, wastewater) and as a resource producer (food, $\left.\mathrm{O}_{2}\right)$. However, this does not preclude the inclusion of other food systems or technologies that are developed to a point where they may trade better or equally as the whole or part of the food and life support system. In fact, we hope this roadmap helps the strategies of researchers with that goal.

Substantial ground research has been done with food crops intended for spaceflight, and this research has provided important data in areas such as water usage, oxygen generation, and other volatile generation. The same research has already benefitted controlled environment agriculture on Earth where it has made important contributions both with LED lighting and early demonstrations of vertical farming [35].

The continuing research both on Earth and in spaceflight to recycle more water and nutrients and provide fresh food sources with substantially less land mass and less intensive agricultural techniques may introduce advances that benefit both Earth and space exploration [44,45]. For example, crop systems have the potential to recycle fertilizer components, such as nitrogen currently reliant on the energy intensive Haber-Bosch system, and potassium and phosphorus reliant on mining, using inedible biomass [46,47] and/or urine $[48,49]$. Although there may be differences in system requirements between Earth and spaceflight, advances could benefit both. However, major challenges remain to be solved with nutrient balances and efficiencies that could cause failures or increase costs in the tightly controlled environments within spacecraft. One long-standing concern for using crops to recycle nutrients from urine is the sodium content, which is not needed by plants and so causes osmotic stress or toxicity [49-51]. Processing and safety protocols that would require additional resources would also need to be established before the use of any wastewater could be considered for food crops.

Although data gathered from crops can vary by type and by system, they provide important baselines for comparison with outcomes from technology advancements (e.g., hardware, software, genome edited cultivars) and alternative conditions (e.g., reduced resources, radiation, reduced pressure). This information is important ultimately to the development of full bioregenerative life support systems. Investigations to date indicate that with high light inputs, around $25 \mathrm{~m}^{2}$ of crops is required for the oxygen production for one person, and $40-50 \mathrm{~m}^{2}$ would be required for caloric production for one person [32,33]. Nutritionally balanced and complete diets would require a greater growth area [40]. Hundreds of liters of water would need to be recycled daily. However, these data are not yet translatable to determine efficient integration with flight ready air and water systems and the associated trade where the resources required for a target amount of crops become 
more cost effective and feasible than the prepackaged food system. Some areas, such as total volume, crew time, power requirements, hardware reliability, and safety have not been tracked over extended continuous use [33], leaving important gaps in understanding these systems. Additionally, data to date do not indicate how these systems and the infrastructure they require will become sustainable.

Finally, while impacts from individual spaceflight stressors, such as microgravity, radiation, and reduced pressure have been investigated for some crops [37,52], more research is needed to evaluate the impacts of multiple stressors on plant production. Ultimately, evaluations on space missions will be needed for accurate integrated assessments and validation. A roadmap for crop research, infrastructure development, and cumulative system integration and validation from the ISS to Mars is depicted in Figure 1. A similar roadmap structure could be applied to other bioregenerative systems or the integration of multiple systems.

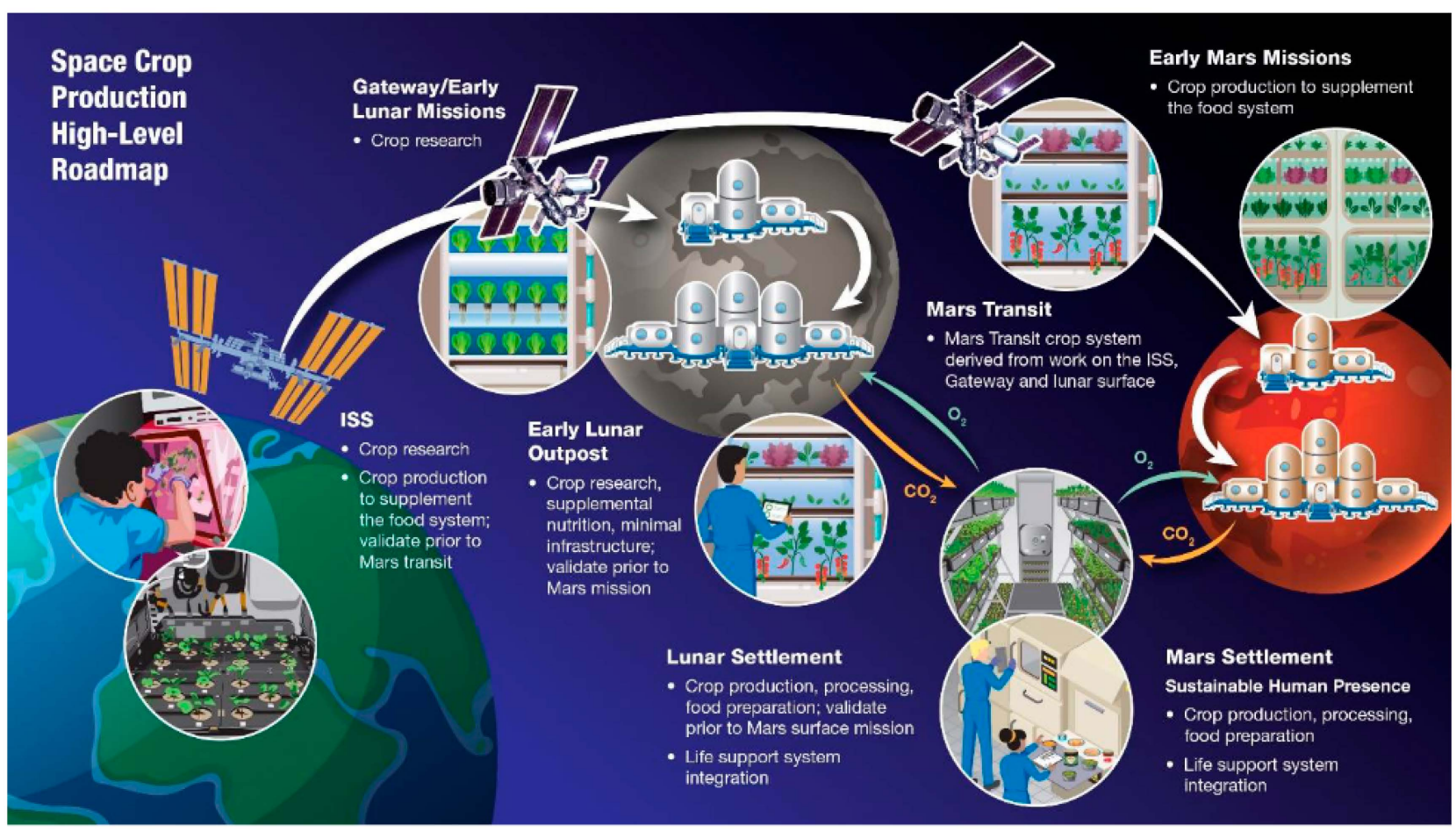

Figure 1. Roadmap for crop research, infrastructure development, and cumulative system integration and validation from the ISS to Mars. A similar roadmap structure could be applied to other bioregenerative systems or integration of multiple systems.

\subsection{Crops for Early Lunar Missions}

Early lunar missions may have limited capabilities for plant growth or sample return to Earth. Some experiments may even be conducted on un-crewed vehicles. Experimental payload capability is currently undefined but may provide opportunities to investigate fundamental questions regarding plant biology in this new deep space environment and validate ground-tested technologies such as autonomous sensing capabilities (multispectral/hyperspectral imaging) that support real-time adjustments to optimize crop health and production [53]. These data may either confirm ground-based data or indicate issues with crop candidates or infrastructure, for example, during seed storage or watering and nutrient delivery under radiation, partial gravity, and reduced pressure environments, that identify research needs. Given the limited research opportunities, we recommend that studies be done to validate ground results from crops rather than with model plant systems so that results can be immediately applied to establish a supplemental food system as missions become longer. 


\subsection{Crops for Early Lunar Outposts}

As mentioned, food preparation infrastructure is expected to be limited on early lunar outpost missions, and therefore, the focus will likely remain on mostly pick-and-eat type salad crops. An early use of scalable, evolvable crop systems could begin to provide continuous growth data that would help demonstrate the requirements for crops in supplemental food production. Ground-based studies to date have identified unpredicted maintenance requirements [54] and differences between predicted and actual productivity [40] that demonstrate the importance of confirming experimental and estimated results under realistic conditions.

This phase may also have sample return, which would enable ground nutritional and microbiological testing to validate nutritional outcomes and food safety protocols. Improved infrastructure or crop selection resulting from the first phase of lunar mission testing may also be validated here prior to the deployment of more advanced systems that may be required as the outpost expands. Over time, the variety of crops is expected to increase as they are validated for overall performance, palatability, safety, and nutrition, within the available infrastructure and crew time.

Infrastructure and crew time requirements would simultaneously need to increase to incorporate greater food preparation infrastructure. Early infrastructure may include cooking capability in the altered gravity and pressure environment. Later capabilities may begin to incorporate the processing of crops, such as wheat or soy. These are examples where specialists or intensive automation may be required, further increasing the resource requirements. Reports on crop-based food systems to date provide some example menus with initial evaluations of acceptability and comparisons to nutritional requirements, but they do not include processing, preparation, and crew time requirements or acceptability over time $[19,40,55]$, leaving a substantial gap. Processing and preparation infrastructure and integration requirements with flight-ready air and water systems also need to be included in trade comparisons with other systems.

It can be expected that at some point during this period, focus will begin to shift from crop selection and production concepts to the optimization of crop systems as a biological component of a controlled environment life support system. As mentioned previously, research will need to be conducted on a range of other topics important to realizing the full potential for crop systems such as nutrient recycling, waste management, microbial plant interaction, the inclusion of automation and integrated control systems, and integration with the air and water system. Ultimately, research will be needed to identify how to produce each piece of this system sustainably, through recycling or ISRU, with no or limited resupply from Earth.

These crop systems would help to meet the needs of an expanding lunar settlement and also serve as a high-fidelity analog and test case for a future Mars capability.

\subsection{Crops on Initial Mars Missions}

Initial Mars missions will require a food system that remains viable for longer durations than any system currently available. A large part of these missions will keep crews in a microgravity environment. While once again, we can expect primary reliance on pre-packaged foods, the ability to supplement key nutrients toward the later part of the missions may be deemed crucial. Some fresh variety and horticultural activities may also provide important psychosocial benefits given the length, isolation, and confinement of these missions in harsh environments. In this context, small-scale supplemental crop production may need to become a reliable part of the food system to provide crew with the supplemental nutrition they might need to maintain their health and performance, but it would not yet be a component of a more complete bioregenerative life support system [56].

Research conducted on the ISS over the past two decades has shown that plants can be grown successfully in the microgravity environment of space and that the salad crops they produced could be both safe to consume and enjoyed by crews [26,37]. While these early demonstrations were primarily focused on addressing fundamental research questions and 
employed growth methods that could not be described as sustainable, work is underway to develop the first operational crop production system to be deployed in space. This system called Ohalo III is planned for deployment on the ISS within the next five years and will provide data on water delivery and volume optimization concepts [57]. When fully developed, it is intended to serve as a prototype for a future crop production system planned for the Mars transit vehicle. Given the scale of the system, it will not provide data on life support integration such as $\mathrm{CO}_{2}$ use and $\mathrm{O}_{2}$ production or the potential to recover nutrients from the waste stream, but it will provide important data on reliability, continuous growth, safety, and maintenance. Ultimately, these data will need to be validated in the integrated gravity, vehicle pressure, atmosphere, and radiation environment of cislunar space and potentially the lunar surface prior to use on Mars transit.

The expectation is that initial Mars surface stays will be short and not require supplemental food production. However, if a crop production capability is available, it would likely be an adaptation of the transit vehicle system or a derivative based on early lunar crop production.

\subsection{Crops for Lunar or Martian Settlements}

It is expected that food specialists will be among the first settlers of the moon or Mars. As the first settlements are established, the role of plants will need to expand from nutritional supplementation and dietary support to a main source of calories. Dedicated crop growth space will be required, and additional processing and preparation infrastructure will be needed to process stable crops such as wheat and soy. Although this infrastructure may come from concepts previously validated on the lunar surface, the ability to bring supplies from Earth to build and integrate similar infrastructure on Mars will be reduced. Production solutions that incorporate ISRU will be critical. This could include the implementation of 3D printing, robotics, sensors, and genomic technologies that can increase production success, reduce crew time, and ultimately support sustainability. There are still significant gaps in each of these areas, which have been reviewed previously [23].

By this point, if not sooner, we can expect the settlement to incorporate crop growth modules with life support systems as technologies are proven reliable and favored in resource trades. Life support infrastructure may connect the crop growth modules to crew habitat areas but keep them environmentally isolated so that optimal conditions for plant growth can be established. A scalable approach of adding additional crop modules as the demands of the settlement grow would ensure a measured incremental increase in capability.

Within the crop modules, the majority of plant horticulture may be performed through a combination of mechanization and robotics all carefully monitored and controlled by a highly capable AI operating system that acts in the role of plant scientist, chemist, and farmer. It is envisioned that the initial crop systems for the settlement will be based on the latest hydroponic growing methods developed for use on Earth [58]. The question frequently arises as to the potential role of Mars regolith as part of ISRU in future crop production. Research is currently underway using a variety of regolith simulants, but further research into both the remediation and augmentation of regolith will be required before we could best address if and how regolith could be incorporated as a soil for plant growth [59].

With incorporation into life support, the challenges become more complex. For example, the balance in these closed systems between $\mathrm{CO}_{2}$ generation from human and plant waste and $\mathrm{O}_{2}$ production must be carefully maintained. A sustainable system would require reintroduction of the plant waste biomass, but within a closed and limited system, the $\mathrm{CO}_{2}$ from biomass oxidation with the $\mathrm{CO}_{2}$ from the humans has the potential to exceed system capacity, and it may require some continued use of physical/chemical methods to achieve full balance $[32,60,61]$. Additional infrastructure for handling plant waste and capabilities for carbon sequestration for things such as soil generation would be needed. 


\section{Considerations for Food Scarcity}

As we move toward bioregenerative systems, we must ensure the prevention of food scarcity from the loss of a system through ways to preserve and store food surplus. Animal protein sources may also continue to be required to support nutritional variety and acceptability for all settlers. As mentioned, even as these systems become less Earth dependent, there may be some supplies still required from Earth. Some prepackaged foods may either need to be continued to be supplied from Earth, or processing infrastructure and capability will be required on Mars to produce them from bioregenerative food surplus. On Earth, within our busy and highly technical lives, we have many food options to ensure caloric availability, nutritional adequacy, and enjoyment. Ready-to-eat foods will continue to be needed on Mars to support activities where time and access to food preparation infrastructure is limited. The successful and healthy exploration and settlement of space may be best served by many food possibilities.

\section{Conclusions}

Readily available safe, nutritious, and acceptable food is critical for human health and performance in spaceflight and on Earth. As missions become longer and further from Earth, food must become more sustainable and less dependent on Earth. Bioregenerative food systems have the potential to provide these capabilities but require significant development to determine feasibility and resource requirements that inform trades between systems and the provision of supplies from Earth. Planned NASA lunar and Mars missions may provide the opportunity to begin collecting data and integrating bioregenerative food systems through a phased approach of system validation that supports increasing caloric and nutritional contributions. It is expected that the technology development and efficiencies gained from this research will similarly benefit sustainability goals on Earth.

Author Contributions: Conceptualization, G.L.D.; funding acquisition, G.L.D.; investigation, G.L.D., R.M.W. and R.F.F.; writing-original draft preparation, G.L.D., R.M.W. and R.F.F.; writing-review and editing, G.L.D., R.M.W. and R.F.F.; All authors have read and agreed to the published version of the manuscript.

Funding: Support for this manuscript was provided by NASA Advanced Exploration Systems.

Institutional Review Board Statement: Not applicable.

Informed Consent Statement: Not applicable.

Data Availability Statement: Data sharing not applicable.

Acknowledgments: We thank Cindy Bush for the graphic and Gioia Massa for critical review of the manuscript.

Conflicts of Interest: The authors declare no conflict of interest.

\section{References}

1. Pimentel, L. Scurvy: Historical review and current diagnostic approach. Am. J. Emerg. Med. 2003, 21, 328-332. [CrossRef]

2. Feeney, R.E.; Houston, C.S. Polar journeys: The role of food \& nutrition in early exploration. Arctic 1998, 51, 386.

3. Douglas, G.L.; Cooper, M.; Bermudez-Aguirre, D.; Sirmons, T. Risk of Performance Decrement and Crew Illness Due to an Inadequate Food System; National Aeronautics and Space Administration (NASA): Houston, TX, USA, 2016.

4. Perchonok, M.H.; Cooper, M.R.; Catauro, P.M. Mission to Mars: Food production and processing for the final frontier. Annu. Rev. Food Sci. Technol. 2012, 3, 311-330. [CrossRef]

5. Smith, S.; Zwart, S.; Kloeris, V.; Heer, M. Nutritional Biochemistry of Space Flight; Nova Science Publishers: New York, NY, USA, 2009.

6. Smith, S.M.; Zwart, S.R.; Douglas, G.L.; Heer, M. Human Adaptation to Spaceflight: The Role of Food and Nutrition, 2nd ed.; NP-2021-03-003-JSC; National Aeronautics and Space Administration Lyndon B. Johnson Space Center: Houston, TX, USA, 2021.

7. Carter, L.; Williamson, J.; Brown, C.; Bazley, J.; Gazda, D.; Schaezler, R.; Tomas, F.; Molina, S. Status of ISS Water Management and Recovery. In Proceedings of the 49th International Conference on Environmental Systems, Boston, MA, USA, 7-11 July 2019. 
8. Landon, L.B.; Douglas, G.L.; Downs, M.E.; Greene, M.R.; Whitmire, A.M.; Zwart, S.R.; Roma, P.G. The Behavioral Biology of Teams: Multidisciplinary Contributions to Social Dynamics in Isolated, Confined, and Extreme Environments. Front. Psychol. 2019, 10, 2571. [CrossRef]

9. Stuster, J. Behavioral Issues Associated with Long-Duration Space Expeditions: Review and Analysis of Astronaut Journals: Experiment 01-E104 (Journals); National Aeronautics and Space Administration, Johnson Space Center: Houston, TX, USA, 2010.

10. Douglas, G.L.; Zwart, S.R.; Smith, S.M. Space food for thought: Challenges and considerations for food and nutrition on exploration missions. J. Nutr. 2020, 150, 2242-2244. [CrossRef]

11. Cooper, M.; Perchonok, M.; Douglas, G.L. Initial assessment of the nutritional quality of the space food system over three years of ambient storage. NPJ Microgravity 2017, 3, 17. [CrossRef]

12. Catauro, P.M.; Perchonok, M.H. Assessment of the long-term stability of retort pouch foods to support extended duration spaceflight. J. Food Sci. 2012, 77, S29-S39. [CrossRef] [PubMed]

13. Nangle, S.N.; Wolfson, M.Y.; Hartsough, L.; Ma, N.J.; Mason, C.E.; Merighi, M.; Nathan, V.; Silver, P.A.; Simon, M.; Swett, J. The case for biotech on Mars. Nat. Biotechnol. 2020, 38, 401-407. [CrossRef] [PubMed]

14. Helisch, H.; Juy-Kieu, C.; Fasoulas, S.; Lapierre, F.; Heyar, A.G. Close the gap-Potential of microalgal biomass for closed ECLSS and future in-situ resource utilization in space. In Proceedings of the 49th International Conference on Environmental Systems, Boston, MA, USA, 7-11 July 2019. ICES-2019-139.

15. Becker, E.W. Microalgae for human and animal nutrition. In Handbook of Microalgal Culture: Applied Phycology and Biotechnology; John Wiley and Sons, Ltd.: Hoboken, NJ, USA, 2013; pp. 461-503.

16. Escobar, C.; Nabity, J. Past, present, and future of closed human life support ecosystems-a review. In Proceedings of the 47th International Conference on Environmental Systems, Boston, MA, USA, 16-20 July 2017.

17. Munns, D.P.; Nickelsen, K. To live among the stars: Artificial environments in the early space age. Hist. Technol. 2017, 33, 272-299. [CrossRef]

18. Manukovsky, N.; Kovalev, V.; Somova, L.; Gurevich, Y.L.; Sadovsky, M. Material balance and diet in bioregenerative life support systems: Connection with coefficient of closure. Adv. Space Res. 2005, 35, 1563-1569. [CrossRef] [PubMed]

19. Fu, Y.; Guo, R.; Liu, H. An optimized 4-day diet meal plan for 'Lunar Palace 1'. J. Sci. Food Agric. 2019, 99, 696-702. [CrossRef]

20. Rubio, N.R.; Xiang, N.; Kaplan, D.L. Plant-based and cell-based approaches to meat production. Nat. Commun. 2020, 11, 6276. [CrossRef] [PubMed]

21. Katayama, N.; Ishikawa, Y.; Takaoki, M.; Yamashita, M.; Nakayama, S.; Kiguchi, K.; Kok, R.; Wada, H.; Mitsuhashi, J.; Force, S.A.T. Entomophagy: A key to space agriculture. Adv. Space Res. 2008, 41, 701-705. [CrossRef]

22. Walford, R.L.; Mock, D.; Verdery, R.; MacCallum, T. Calorie restriction in biosphere 2: Alterations in physiologic, hematologic, hormonal, and biochemical parameters in humans restricted for a 2-year period. J. Gerontol. Ser. A Biol. Sci. Med. Sci. 2002, 57, B211-B224. [CrossRef] [PubMed]

23. Anderson, M.S.; Barta, D.; Douglas, G.; Motil, B.; Massa, G.; Fritsche, R.; Quincy, C.; Romeyn, M.; Hanford, A. Key gaps for enabling plant growth in future missions. In Proceedings of the AIAA SPACE and Astronautics Forum and Exposition, Orlando, FL, USA, 12 October 2017.

24. Keeble, B.R. The Brundtland report:'Our common future. Med. War 1988, 4, 17-25. [CrossRef]

25. Rahman, A.; Galazka, J.; Dougherty, M.; Jones, H.; Hogan, J. Methane as a Carbon Substrate for Biomanufacturing. In Proceedings of the 48th International Conference on Environmental Systems, Boston, MA, USA, 8-12 July 2018. ICES-2018-193.

26. Massa, G.D.; Dufour, N.F.; Carver, J.A.; Hummerick, M.E.; Wheeler, R.M.; Morrow, R.C.; Smith, T.M. VEG-01: Veggie hardware validation testing on the International Space Station. Open Agric. 2017, 2, 33-41. [CrossRef]

27. Malhotra, A.; Redberg, R.F.; Meier, P. Saturated fat does not clog the arteries: Coronary heart disease is a chronic inflammatory condition, the risk of which can be effectively reduced from healthy lifestyle interventions. Br. J. Sports Med. 2017, 51, 1111-1112. [CrossRef]

28. De Souza, R.J.; Mente, A.; Maroleanu, A.; Cozma, A.I.; Ha, V.; Kishibe, T.; Uleryk, E.; Budylowski, P.; Schünemann, H.; Beyene, J. Intake of saturated and trans unsaturated fatty acids and risk of all cause mortality, cardiovascular disease, and type 2 diabetes: Systematic review and meta-analysis of observational studies. BMJ 2015, 351, h3978. [CrossRef]

29. Sirmons, T.A.; Roma, P.G.; Whitmire, A.M.; Smith, S.M.; Zwart, S.R.; Young, M.; Douglas, G.L. Meal replacement in isolated and confined mission environments: Consumption, acceptability, and implications for physical and behavioral health. Physiol. Behav. 2020, 219, 112829. [CrossRef]

30. Douglas, G.; Johnson, M.; Broyan, J. Space food system water content: Considerations for ECLSS water system closure. In Proceedings of the 50th International Conference on Environmental Systems, Boston, MA, USA, 12-15 July 2021. ICES-2021-130.

31. Burgner, S.E.; Nemali, K.; Massa, G.D.; Wheeler, R.M.; Morrow, R.C.; Mitchell, C.A. Growth and photosynthetic responses of Chinese cabbage (Brassica rapa L. cv. Tokyo Bekana) to continuously elevated carbon dioxide in a simulated Space Station "Veggie" crop-production environment. Life Sci. Space Res. 2020, 27, 83-88. [CrossRef]

32. Wheeler, R.M. Carbon balance in bioregenerative life support systems: Some effects of system closure, waste management, and crop harvest index. Adv. Space Res. 2003, 31, 169-175. [CrossRef]

33. Fu, Y.; Li, L.; Xie, B.; Dong, C.; Wang, M.; Jia, B.; Shao, L.; Dong, Y.; Deng, S.; Liu, H. How to establish a Bioregenerative Life Support System for long-term crewed missions to the Moon or Mars. Astrobiology 2016, 16, 925-936. [CrossRef] [PubMed] 
34. Zabel, P.; Zeidler, C.; Vrakking, V.; Dorn, M.; Schubert, D. Biomass production of the EDEN ISS space greenhouse in Antarctica during the 2018 experiment phase. Front. Plant Sci. 2020, 11, 656. [CrossRef] [PubMed]

35. Wheeler, R.M. Agriculture for space: People and places paving the way. Open Agric. 2017, 2, 14-32. [CrossRef]

36. Vrakking, V.; Schubert, D.; Zabel, P.; Zeidler, C.; Dorn, M.; Ferl, R.; Paul, A.-L. 4.12 EDEN ISS-greenhouse in Antarctica. In Expeditions to Antarctica: ANT-Land 2019/20 Neumayer Station III, Kohnen Station, Flight Operations and Field Campaigns; DLR: Bremen, Germany, 2020; p. 54.

37. Sychev, V.; Levinskikh, M.; Gurieva, T.; Podolsky, I. Biological life support systems for space crews: Some results and prospects. Hum. Physiol. 2011, 37, 784-789. [CrossRef]

38. Gitelson, I.; Terskov, I.; Kovrov, B.; Sidko, F.Y.; Lisovsky, G.; Okladnikov, Y.N.; Belyanin, V.; Trubachov, I.; Rerberg, M. Life support system with autonomous control employing plant photosynthesis. Acta Astronaut. 1976, 3, 633-650. [CrossRef]

39. Hanford, A. Advanced Life Support Baseline Values and Assumptions Document JSC 47804; NASA JSC: Houston, TX, USA, 2002.

40. Masuda, T.; Arai, R.; Komatsubara, O.; Tako, Y.; Harashima, E.; Nitta, K. Development of a 1-week cycle menu for an Advanced Life Support System (ALSS) utilizing practical biomass production data from the closed ecology experiment facilities (CEEF). Habitation 2005, 10, 87-97. [CrossRef]

41. Drysdale, A.; Ewert, M.; Hanford, A. Equivalent System Mass Studies of Missions and Concepts; SAE International: Warrendale, PA, USA, 1999.

42. Drysdale, A.; Maxwell, S.; Ewert, M.; Hanford, A. Systems Analysis of Life Support for Long-Duration Missions; Soc Automotive Eng Technical Paper 2000-01-2394; SAE: Warrendale, PA, USA, 2000.

43. Odeh, R.; Guy, C.L. Gardening for therapeutic people-plant interactions during long-duration space missions. Open Agric. 2017, 2,1-13. [CrossRef]

44. Van Ginkel, S.W.; Igou, T.; Chen, Y. Energy, water and nutrient impacts of California-grown vegetables compared to controlled environmental agriculture systems in Atlanta, GA. Resour. Conserv. Recycl. 2017, 122, 319-325. [CrossRef]

45. Wheeler, R.; Sager, J.; Mackowiak, C.; Stutte, G.; Yorio, N.; Ruffe, L.; Berry, W. Nutrient, acid and water budgets of hydroponically grown crops. Acta Hortic. 1999, 481, 655-661. [CrossRef]

46. Mackowiak, C.; Garland, J.; Strayer, R.; Finger, B.; Wheeler, R. Comparison of aerobically-treated and untreated crop residue as a source of recycled nutrients in a recirculating hydroponic system. Adv. Space Res. 1996, 18, 281-287. [CrossRef]

47. Lunn, G.; Stutte, G.; Spencer, L.; Hummerick, M.; Wong, L.; Wheeler, R. Recovery of nutrients from inedible biomass of tomato and pepper to recycle fertilizer. In Proceedings of the 47th International Conference on Environmental Systems, Boston, MA, USA, 16-20 July 2017.

48. El-Nakhel, C.; Geelen, D.; De Paepe, J.; Clauwaert, P.; De Pascale, S.; Rouphael, Y. An Appraisal of Urine Derivatives Integrated in the Nitrogen and Phosphorus Inputs of a Lettuce Soilless Cultivation System. Sustainability 2021, 13, 4218. [CrossRef]

49. Deng, S.; Xie, B.; Liu, H. The recycle of water and nitrogen from urine in bioregenerative life support system. Acta Astronaut. 2016, 123, 86-90. [CrossRef]

50. Zabel, P.; Bornemann, G.; Tajmar, M.; Schubert, D. Yield of dwarf tomatoes grown with a nutrient solution based on recycled synthetic urine. Life Sci. Space Res. 2019, 20, 62-71. [CrossRef]

51. Mackowiak, C.; Grossl, P.; Wheeler, R. Crop effects on closed system element cycling for human life support in space. J. Plant Nutr. 2009, 32, 318-334. [CrossRef]

52. Corey, K.A.; Barta, D.J.; Wheeler, R.M. Toward Martian agriculture: Responses of plants to hypobaria. Life Support Biosph. Sci. 2002, 8, 103-114.

53. Monje, O.; Nugent, M.; Hummerick, M.; Dreschel, T.; Spencer, L.; Romeyn, M.; Massa, G.; Wheeler, R.; Fritsche, R. New frontiers in food production beyond LEO. In Proceedings of the 49th International Conference on Environmental Systems, Boston, MA, USA, 7-11 July 2019.

54. Bunchek, J.; Curry, A.; Romeyn, M. Sustained veggie: A continuous food production comparison. In Proceedings of the 50th Interntaional Conference on Environmental Systems, Boston, MA, USA, 12-15 July 2021.

55. Cooper, M.R.; Catauro, P.; Perchonok, M. Development and evaluation of bioregenerative menus for Mars habitat missions. Acta Astronaut. 2012, 81, 555-562. [CrossRef]

56. Kliss, M.; Heyenga, A.; Hoehn, A.; Stodieck, L. Recent advances in technologies required for a "Salad Machine". Adv. Space Res. 2000, 26, 263-269. [CrossRef]

57. Wheeler, R.; Massa, G. Ohalo III, The First Operational Crop Production System and Protocype for a Mars Transit Vehicle (Ohalo). 2021. Available online: https:// techport.nasa.gov/view/97036 (accessed on 1 March 2021).

58. Jones, J.B., Jr. Complete Guide for Growing Plants Hydroponically; CRC Press: Boca Raton, FL, SUA, 2014.

59. Cannon, K.M.; Britt, D.T.; Smith, T.M.; Fritsche, R.F.; Batcheldor, D. Mars global simulant MGS-1: A Rocknest-based open standard for basaltic martian regolith simulants. Icarus 2019, 317, 470-478. [CrossRef]

60. Tako, Y.; Arai, R.; Tsuga, S.-I.; Komatsubara, O.; Masuda, T.; Nozoe, S.; Nitta, K. CEEF: Closed ecology experiment facilities. Gravit. Space Res. 2010, 23, 321-338.

61. Wheeler, R.M. Gas balance in a plant-based CELSS. In Plants in Space Biology; Suge, H., Ed.; Institute of Genetic Ecology, Tohoku University Press: Tohoku, Japan, 1996. 